\title{
UN CAND|DATO per le medaglie Fields
}

di Renato Betti

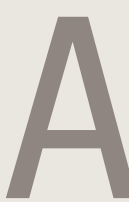

L Congresso internazionale dei matematici che si terrà in agosto a Rio de Janeiro verranno attribuite le medaglie Fields, premio destinato ad un massimo di quattro matematici di età inferiore ai 40 anni. Il riconoscimento, assegnato ogni quattro anni fin dal 1936, ha lo stesso valore simbolico del premio Nobel (com'è noto mancante per la Matematica) con l'ulteriore e significativa restrizione della giovane età. Quest'anno, nei "pronostici" di tutti ricorre come possibile vincitore un candidato che, con i suoi 30 anni, sarebbe uno dei più giovani di sempre: Peter Scholze, già da sei anni docente all'Università di Bonn. Le motivazioni di altri premi che gli sono stati attribuiti nel frattempo parlano di "contributi rivoluzionari in numerosi campi al confine fra Geometria aritmetica, algebrica e Teoria delle forme automorfe" (premio Ramanujan, assegnato a matematici più giovani di 32 anni, l'età in cui Ramanujan è morto) o anche di "uno dei rari talenti che emergono soltanto dopo decenni" (premio Leibniz, assegnato dalla Fondazione di Ricerca Tedesca (DFG) a "scienziati e accademici per i loro eccezionali risultati di ricerca", ricevuto da Scholze nel 2016).

Dalla motivazione del premio Leibniz, leggiamo: "In anni recenti ha risposto a questioni fondamentali di Geometria aritmetica e algebrica rimaste irrisolte per decenni. Ciò è in particolare vero per la sua dimostrazione della cosiddetta congettura di Langlands per i corpi locali p-adici. La sua teoria dei cosiddetti spazi perfettoidi ha enormemente esteso lo spettro dei metodi matematici".

L'idea fondamentale di Scholze, su cui ha cominciato a lavorare ed a produrre risultati quand'era ancora studente, riguarda la classe di spazi che ha chiamato "perfettoidi", il cui significato occorre all'intersezione fra Geometria algebrica e Teoria dei numeri.

La sua costruzione ha origine nel sistema dei numeri $p$-adici, numeri che, per ogni numero primo $p$, forniscono una diversa nozione di "valore assoluto": due numeri $p$-adici sono tanto più prossimi quanto più alta è la potenza di $p$ per cui è divisibile la loro differenza. In questo modo, la nozione incorpora profonde informazioni sulla relazione di congruenza modulo $p$, con applicazione, ad esempio, alla dimostrazione dell'ultimo teorema di Fermat, da parte di Andrew Wiles, e possibilmente all'ipotesi di Riemann, l'affascinante congettura sulla distribuzione dei numeri primi.

Il punto fondamentale consiste nel rappresentare un numero primo con una "torre" infinita di numeri $p$-adici associati e rappresentarlo come variabile di un'equazione, semplificando l'Aritmetica grazie all'uso di oggetti più complessi e rendendo possibile l'applicazione di metodi geometrici in contesti aritmetici.

Senza scomodare la Geometria analitica di René Descartes, è noto che larga parte della Matematica, fin dai tempi più antichi, si è svolta proprio nel rapporto fra gli aspetti qualitativi, relativi alle forme geometriche e le loro trasformazioni, e quelli quantitativi dell'Aritmetica, dei numeri e della manipolazione di simboli astratti. Al punto, "ciò che non ha parti", l'elemento primario della Geometria euclidea, viene associato un dato numerico elementare ed essenziale. Così le curve si trasformano in equazioni, le coordinate possono variare in domini numerici diversi e tutta la Matematica prende uno sviluppo inaspettato. Gli

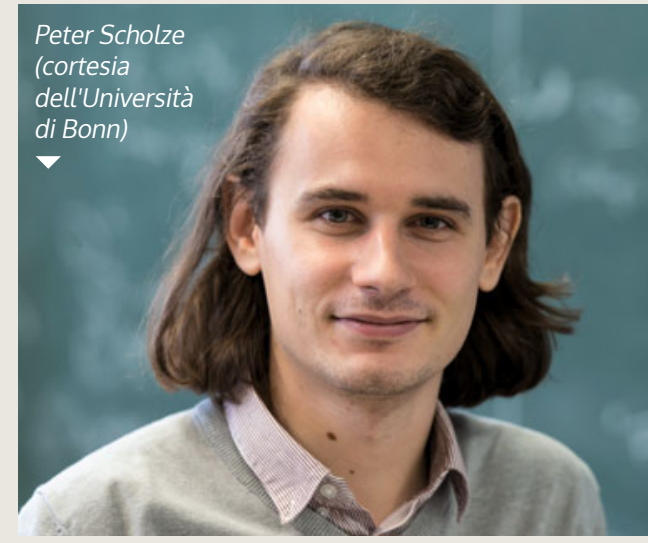

stessi numeri $p$-adici, motivati originariamente dal desiderio di applicare le tecniche delle serie di potenze alla Teoria dei numeri, estendono la propria influenza all'Analisi matematica. La Geometria aritmetica, in cui la Teoria dei numeri si collega alla Geometria, nasce dal tentativo di Diofanto di studiare le soluzioni intere delle equazioni polinomiali con i metodi della Geometria algebrica. Scholze parte proprio da qui e viene subito coinvolto in varie strutture che percorrono numerose aree della Matematica: curve ellittiche, forme modulari e altre nozioni dal tono esotico. Presto, con la tesi di dottorato del 2012, risolve una complicata congettura relativa alle soluzioni $p$-adiche dei polinomi, nota come "weight monodromy", enunciata nel 1978 da Pierre Deligne. L'anno successivo ottiene un risultato sulle leggi di reciprocità dell'Aritmetica modulare con il quale interagisce ed estende il programma con cui il matematico Robert Langlands tende a mettere in relazione la Teoria dei numeri con l'Analisi armonica. Programma che oggi è riconosciuto come il maggior tentativo di unificazione dei principali settori in cui si articola la Matematica: Geometria, Aritmetica ed Analisi.

Il lavoro continua con successo ed ammirazione: Robert Langlands ha vinto il prestigioso premio Abel, un premio che viene assegnato ogni anno "alla carriera", con la motivazione: "Per il suo visionario programma che collega la Teoria della rappresentazione alla Teoria dei numeri". La concezione visionaria di una grande teoria unitaria della Matematica prosegue con il lavoro di Scholze il quale, dicono gli esperti, mostra fra l'altro che il programma di Langlands è più originale, profondo e sistematico di quanto si pensava. 